\title{
Coronary Sinus Cryoablation of Ventricular Tachycardia after Failed Radiofrequency Ablation
}

\author{
Jiménez-Fernández M*, Macías- Ruiz R, Álvarez-López M, and Tercedor- Sánchez L
}

Virgen de las Nieves University Hospital, Granada, Spain

Received: March 11, 2014; Accepted: September 10, 2014; Published: September 25, 2014

*Corresponding author: Miriam Jiménez-Fernández, Virgen de las Nieves University Hospital, Granada, Spain, E-mail: mirijf84@hotmail.com

\section{Abstract}

We present a case of Great Cardiac Vein (GCV) cryoablation in order to suppress idiopathic epicardial Ventricular Tachycardia (VT) after failed Radiofrequency (RF) ablation via the epicardium and GCV. RF ablation is the technique of choice for the treatment of Ventricular Arrhythmias (VA). However, this procedure has its limitations when delivered inside the distal cardiac veins. Cryoablation is a good alternative in patients where RF cannot be used because of risk of high impedance.
\end{abstract}

Keywords: Percutaneous epicardial ablation; Ventricular tachycardia; Radiofrequency; Catheter ablation; Cryoablation; Coronary sinus; High impedance; Cryoenergy; Great cardiac vein; Mapping; Electroanatomic mapping system; 12-lead surface electrocardiogram

\section{Case Report}

A 56-year-old female patient presented in emergency room referring shortness of breath and chest pain. ECG showed sustained monomorphic VT at 160 beats per minute with right bundle branch block and right inferior axis morphology (Figure $1 \mathrm{~A}$ ), requiring electrical cardioversion due to haemodynamic instability. The patient was admitted to the hospital. Physical exam, blood tests and 12-lead ECG during sinus rhythm were normal; the transthoracic echocardiography showed mild hypertensive heart disease. Several runs of no sustained VT and ventricular premature contractions with the same morphology as the sustained VT repeated the following days, despite treatment with bisoprolol, so we decided to perform a catheter ablation.

In the first Endocardial Electro Physiological (EP) study, the voltage map of the Endocardial Left Ventricle (LV), made with Carto 3 system (Biosense Webster, Diamond Bar, CA), revealed no scarring and there was no pace-map lead match. An epicardial origin of VT was suspected, so ablation was not carried out.

The second EP study was performed four days later, using a subxiphoid epicardial approach. Activation mapping was consistent with a focal origin at the basal anterior segment of the LV (Figure 1B), with a 12/12 pace-map lead match. RF ablation with an irrigated-tip catheter at $50 \mathrm{~W}$ suppressed the VT but it recurred after 72 hours.

In a third EP five days later we approached the epicardium via the coronary sinus. Mapping showed earliest activation (-28 $\mathrm{ms})$ on the distal GCV, close to the origin of the anterior interventricular vein, and near to the previous epicardial ablation zone, with a $12 / 12$ pace-map lead match in this area. RF with an open-tip $3.5 \mathrm{~mm}$ irrigated catheter momentarily suppressed the tachycardia, but the delivery of RF had to be interrupted with a maximal energy of $15 \mathrm{~W}$, as the impedance rise exceeded the safety limit (>300 $\Omega$ ) (Figure 1C). In order to solve this problem, we decided to change to a $6 \mathrm{~mm}$ tip cryoablation catheter that was able to reach the programmed temperature of $-80^{\circ} \mathrm{C}$ suppressing the VT with no further recurrences. An echocardiogram showed no pericardial effusion and the patients remained asymptomatic without Ventricular Arrhythmias in Holter monitoring at 1, 6 and 12 months after the procedure.

\section{Discussion}

Catheter ablation of VA originating from near the GCV is technically demanding and it frequently needs an Epicardial approach. Epicardial mapping and ablation in the GCV area are limited due to the presence of fat and the overlying left atrial appendage and coronary arteries [1].

Catheter ablation with RF from inside the cardiac veins is an effective technique in the treatment of several arrhythmias, when endocardial or percutaneous epicardial approach is not successful. But the application of this energy inside the GCV is related with potential severe complications, such as cardiac perforation or damage of epicardial structures like coronary arteries or phrenic nerves [2].

$\mathrm{RF}$ ablation with open-irrigated catheters is the current gold standard technique for VT ablation, as it is able to create larger and deeper lesions compared with cryoenergy. However, there are some reports of ineffectiveness of RF application inside distal GCV due to high impedance, which makes impossible to deliver enough amount of radiofrecuency energy to suppress the tachycardia [2].

Cryoablation may be a useful alternative in these cases, as 


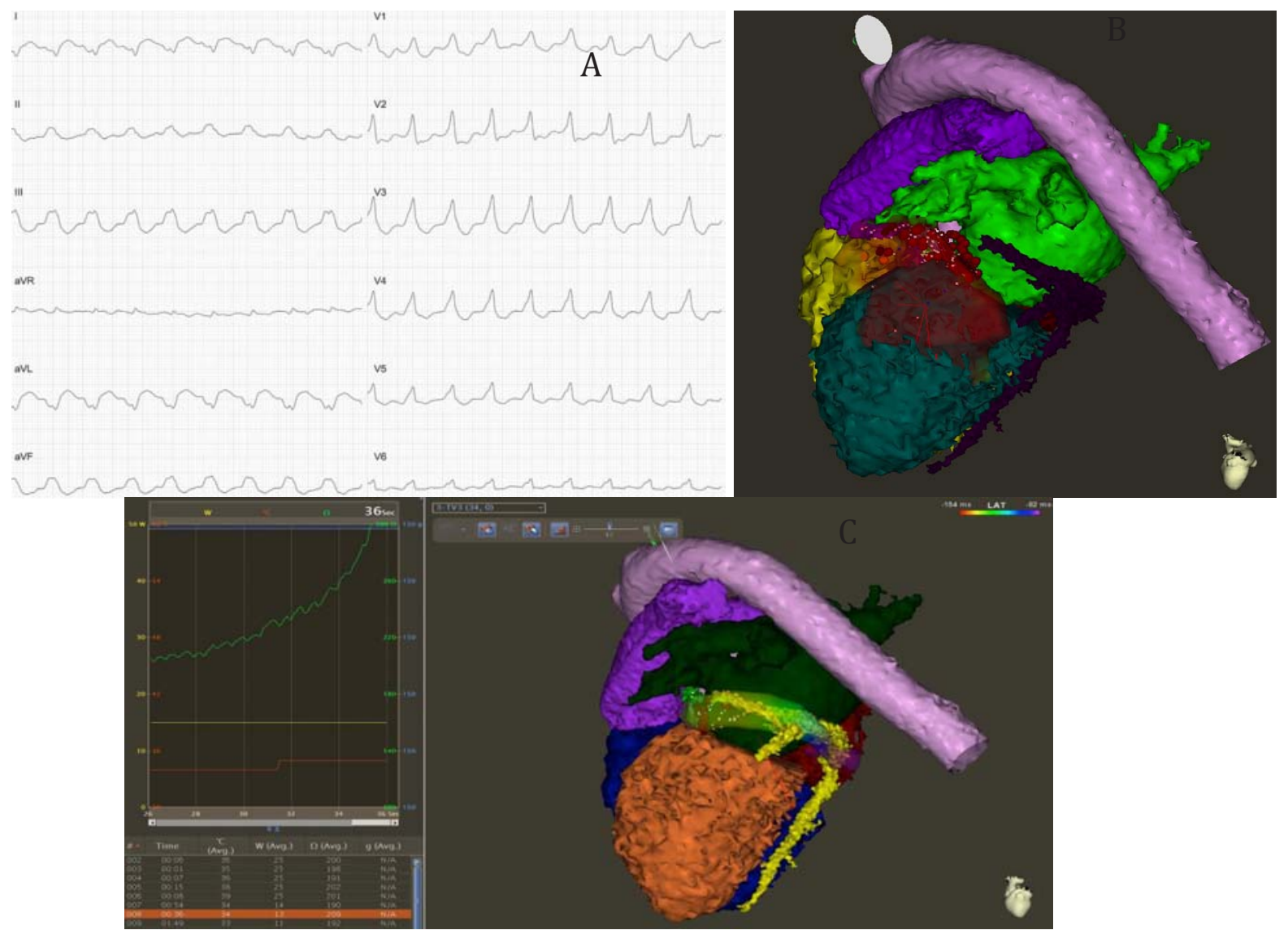

Figure 1: A) Twelve-lead electrocardiogram of the sustained monomorphic VT at 160 beats per minute with right bundle branch block and right inferior axis morphology. B) Anatomical picture obtained with the integration of CT angiography with the epicardial map using the Carto 3 system (Biosense Webster, Diamond Bar, CA). The epicardial map has been partially removed to show the application of RF energy (red tag). AO (Aorta), PA (Pulmonary Artery), RVOT (Right Ventricle Outflow Tract), LA (Left Atrium), LV (Left Ventricule), GCV (Great Cardiac Vein). C) On the right we can see the Carto 3 imaging obtained during the third EP study, resulting from the integration of the CT angiography with the map of the GCV. Red tag represents RF application. On the left graph the impedance rise is shown with a green line (white arrow), temperature in a yellow line and watts in orange during a RF application.

there is a lower risk of damaging the coronary arteries or causing heart rupture, the lesions are not as deep and the tissue is better preserved than when RF is used [3]. Moreover, it is not hindered by high impedance inside the cardiac veins and is likely facilitated by low blood flow. However, the cryocatheter is stiffer and it can be difficult to manoeuvre in the coronary venous system.

Our case report shows that cryoenergy may be an effective and safer alternative against RF when ablating potential life threatening epicardial ventricular arrhythmias from inside the distal GCV, as it does not depend on impedances levels.

\section{References}

1. Yamada T, McElderry H, Doppalapudi H, Okada T, Murakami Y, Yoshida
Y, et al. Idiopathic ventricular arrhythmias originating from the left ventricular summit: anatomic concepts relevant to ablation. Circ Arrhythm and Electrhophysiol. 2010; 3(6): 616-623. doi: 10.1161/ CIRCEP.110.939744.

2. Obel OA, d'Avila A, Neuzil P, Saad EB, Ruskin JN, Reddy VY. Ablation of left ventricular epicardial outflow tract tachycardia from the distal great cardiac vein. J Am Coll Cardiol. 2006 Nov 7; 48(9): 1813-1817.

3. Di Biase L, Al-Ahmad A, Santanqeli P, Hsia HH, Sanchez J, Bailey S, et al. Safety and outcomes of cryoablation for ventricular tachyarrhythmias: results from a multicenter experience. Heart Rhythm. 2011 Jul; 8(7): 968-974. doi: 10.1016/j.hrthm.2011.02.038. 